\title{
The cardiovascular burden of light smoking
}

\author{
Vasiliki Katsi ${ }^{1}$, Spyridon Maragkoudakis ${ }^{2}$, Nikolaos loakeimidis ${ }^{1}$, Maria Marketou ${ }^{2}$, \\ Fragkiskos Parthenakis ${ }^{2}$, Charalambos Vlachopoulos ${ }^{1}$, Konstantinos Tsioufis ${ }^{1}$
}

${ }^{1}$ First Department of Cardiology, 'Hippokration' Hospital of Athens, Athens, Greece ${ }^{2}$ Cardiology Department, University Hospital of Heraklion, Athens, Greece

Submitted: 2 January 2021

Accepted: 1 March 2021

Arch Med Sci Atheroscler Dis 2021; 6: e48-e56

DOI: https://doi.org/10.5114/amsad.2021.105313

Copyright @ 2021 Termedia \& Banach

\begin{abstract}
The assumption that light cigarette smoking, meaning smoking one to five cigarettes per day, is not so harmful has been dissipated by several studies. Regardless of the quantity of tobacco cigarettes, smoking remains a leading risk factor for the development and progression of cardiovascular diseases. Smoke is a mixture of several toxic chemicals, such as nicotine, carbon monoxide, and oxidants, implicated in the pathogenesis of cardiovascular and pulmonary diseases. Despite anti-smoking campaigns, a misconception concerning "safe smoking" still exists. The purpose of this literature review is to highlight the deleterious effect of light cigarette smoking and claim the consensus that there is no safe smoking.
\end{abstract}

Key words: light smoking, cardiovascular risk, cardiovascular disease.

\section{Introduction}

Cardiovascular disease (CVD) remains a leading cause of morbidity and mortality, despite the considerable advances that have been achieved in preventive and therapeutic strategies. It constitutes the primary cause of death of 3.9 million people in Europe each year, causing $45 \%$ of all deaths in Europe, with the incidence continuously rising due to increased risk factors and ageing [1]. Cigarette smoking is a well-established cardiovascular risk factor, and it has been estimated by the World Health Organization that it accounts for roughly 2.5 million premature deaths attributed to CVD [2].

Currently, despite extensive anti-smoking campaigns, it is estimated that about one billion adults worldwide are still active smokers. Although the prevalence of conventional cigarette smoking in the general population has declined, population growth, as well as persistently high prevalence of smoking in highly populated developing countries, translates into an increasing trend in the absolute number of active smokers [3]. Smoking a few cigarettes, and even just a single cigarette, each day still carries high risk for heart attack and stroke [4].

The principal purpose of this literature review is to evaluate the existing accessible published data regarding the impact of low-grade tobacco smoke exposure on cardiovascular health. We performed a review and examination of existing evidence from clinical trials investigating the relationship between degree of exposure and outcomes, the pathophysiological pathways linking low-grade tobacco smoke exposure to

\author{
Corresponding author: \\ Spyridon Maragkoudakis \\ Cardiology Department \\ University Hospital \\ of Heraklion \\ Athens, Greece \\ E-mail: smaragoudakis79@ \\ hotmail.com
}


cardiovascular risk and the management of light cigarette smokers with regard to eventual cessation. Whereas our primary focus was on active smoking, we also included selected evidence from studies on second-hand smoking, at points where extrapolations were considered appropriate due to relative or absolute paucity of data on different effects of low-grade active smoking.

\section{Quantification of smoking status}

Smoking status is a continuous variable; exposure to tobacco smoke can range from intermittent or occasionally active or second-hand smoking to a daily consumption of more than 40 cigarettes. In addition, it should be viewed as a dynamic rather than a static parameter because smoking status can shift in both directions many times throughout an individual's lifespan. The active tobacco consumption grade can be expressed as number of cigarettes per day (CPD), grams of tobacco per day, or pack years, depending on whether one focuses on short- or long-term effects and on the exact tobacco product consumed. The degree of exposure to second-hand smoke, on the other hand, is more difficult to quantify because the concentrations of harmful substances in inhaled air vary continuously over time, as does the daily total time of exposure. These facts pose significant methodological difficulties on the efforts to precisely define the dose-response relationship between the level of tobacco smoke exposure and the incidence of adverse cardiovascular events.

There is no universal definition for "light smoking". Consumption of less than 1 pack per day, less than 15 cigarettes per day, less than 10 cigarettes per day, and 1 to 5 cigarettes per day have all been used in this context, as well as consumption of cigarettes of low nicotine and tar content $[5,6]$. In any case, the proportion of "light" smokers seems to demonstrate a rising trend. In particular, according to the results of the Health Survey for England, the percentage of smokers consuming 1 to 5 cigarettes per day demonstrated an increased trend from $18.2 \%$ to $23.6 \%$ between 2009 and 2014, while the percentage of smokers who consume less than 10 cigarettes per day increased from $16 \%$ to $27 \%$ between 2005 and 2014 [7]. These data are in agreement with the findings of a similar study conducted in the US, where the proportion of smokers who consume less than 10 cigarettes per day was reported to have increased from 16\% to 27\% between 2005 and 2014 [8]. During the last decades, there has been an accumulation of evidence suggesting an unexpectedly steep rise in cardiovascular risk at low levels of cigarette consumption.

Smoking is highly addictive, and cutting down on the daily number of cigarettes might appear to smokers as a more feasible target as compared to quitting; however, the strength of motivation to cease smoking greatly depends on comprehensive knowledge relevant to the potential impact of persistent "light" smoking on health. In this context, it should be underlined that smoking a few cigarettes per day, along with smoking low-nicotine cigarettes, seems to be regarded as relatively safe by a significant part of the general population. Indeed, one study that was conducted in the United States, evaluating the perception of young individuals with respect to the impact of light or intermittent smoking on health, showed that nearly one quarter of adolescents ignore the potential harmful effects of light and intermittent smoking. More precisely, among 24,658 adolescents, only $35 \%$ considered low-grade tobacco consumption as significantly harmful, while $10 \%$ perceived "light smoking" as harmless [9]. Accordingly, the findings of the Health Survey for England - in which $26 \%$ of current smokers reported that they were willing to reduce tobacco consumption but did not aim to quit - probably reflects a perception that "light" smoking does not pose a significant threat to health [7].

The aforementioned growing proportion and absolute number of "light" smokers, coupled with the strong association between smoking and CVD, highlight the importance of knowledge of the potential impact of low-grade tobacco consumption on cardiovascular health. Given the existing evidence that the relationship between smoking intensity and incidence seems to be approximately linear in the case of lung cancer - as suggested by the findings of the American Cancer Society Prevention Study II [10] - it could be expected that an analogous pattern of dose-response could exist with respect to CVD, as well.

\section{Impact of chronic light smoking on cardiovascular outcomes}

One of the major studies specifically aiming to investigate the health risk associated with an active smoking consumption as low as 1 to 4 cigarettes per day was conducted in Norway by Bjartveit and Tverdal [11] In this prospective study, 23,521 men and 19,201 women aged 35-49 years were enrolled. Participants were screened for cardiovascular risk factors and established CVD from 1972 to 1978 , and follow-up was continued until 2002. Individuals with a history of myocardial infarction, definite or suspected stable coronary artery disease or a history of stroke, diabetes, or peripheral artery disease were excluded, as were former smokers. Smoking status was assessed via questionnaire, whereby the participants could report their daily cigarette consumption either by ticking one of the preset categories (1-4, 5-9, 
$10-14,15-19,20-24,25+$ cigarettes per day) or by direct input of the actual number or range of cigarettes smoked per day in a special box in the questionnaire. For participants providing a range rather than a specific number of cigarettes consumed per day, the highest number of the range was used. In order to achieve maximal consistency while also including subjects consuming hand-rolled cigarettes, the equivalence between factory-made and hand-rolled cigarettes was pre-specified, and study nurses were instructed accordingly. Study endpoints included all-cause mortality and adjusted relative risks of dying from ischaemic heart disease, any cancer, and lung cancer. The findings of this study indicated a relative risk of dying from ischaemic heart disease as high as 2.74 in male smokers consuming 1-4 cigarettes per day and 2.94 in female subject of the same smoking category. Characteristically, the steepest increase in cardiovascular mortality was observed in both sexes at consumption levels between 0 and 1-4 cigarettes per day, while the rise rate tended to be lower at higher levels. It should be mentioned that, although a substantial proportion of "light" smokers had changed smoking category at 10-year follow-up, the number of participants who ceased smoking during the study was comparable to that of subjects that increased consumption.

Additional, albeit indirect, evidence regarding the possibly higher than expected contribution of low-grade smoke consumption to the development of CVD was provided 2 decades ago by the work of Law et al. [12] in a systematic review where exposure to second-hand smoke was found to carry a relative risk of 1.3 for coronary artery disease-associated death. This relative risk was considered disproportionately high, given that early studies evaluating the association between active smoking and CVD reported a hazard ratio value of between 2 and 3 . In support of these findings are the results of a meta-analysis of 5 cohort studies evaluating the dose-exposure association between active smoking and ischaemic heart disease, which suggested that smoking 1 cigarette per day carried a relative risk for developing coronary artery disease compatible with that reported for second-hand smoking [12]. In another interesting approach, Pope et al. [13] provided an estimate of the dose-response relationship between level of tobacco consumption and incidence of adverse cardiovascular events based on combined data on second-hand smoking, light and heavy active smoking, as well as particulate matter from air pollution. The results of their analysis indicated that levels of consumption as low as 4-7 cigarettes per day carry roughly $70 \%$ of the effect of smoking 23 cigarettes daily on cardiovascular health.
In support of the potentially detrimental effect of light smoking on cardiovascular health are the results of a 22-year follow-up of 12,149 subjects enrolled in the Copenhagen City Heart Study one of the largest studies to have investigated the prevalence and impact of various cardiovascular risk factors in the general population [14]. Subjects were enrolled in 1976 and followed-up until 1998, focusing on the incidence of myocardial infarction and all-cause death. Tobacco consumption was expressed in grams rather than cigarettes per day, equating a cigarette to $1 \mathrm{~g}$ a cheroot to $3 \mathrm{~g}$, and a cigar to $5 \mathrm{~g}$ of tobacco. Remarkably, this is - to our knowledge - the only study to have included "non-inhaling" smokers as a separate group. Current tobacco consumption was calculated by equating a cigarette to $1 \mathrm{~g}$, a cheroot to $3 \mathrm{~g}$, and a cigar to $5 \mathrm{~g}$ of tobacco. For women consuming 3-5 g of tobacco daily, the relative risk for myocardial infarction was estimated at 2.14. In men, on the other hand, consumption of 6-9 g daily was estimated as the threshold for a significant increase in cardiovascular risk (relative risk $=2.10$ ), with the exposure-effect curve levelling off at higher consumption levels. Notably, trends toward worse outcomes were also observed in non-inhaling men and women, albeit without reaching statistical significance.

Interestingly, a recent meta-analysis examined the potential impact of tobacco consumption as low as 1 cigarette per day on cardiovascular outcomes in subjects without established CVD [4]. Results of 141 separately conducted cohort studies were analysed. The strength of association between consumption of 1 to 5 cigarettes per day and cardiovascular outcome - besides relative risk - was expressed as the percentage of the excess relative risk carried by smoking 20 cigarettes per day. Accordingly, smoking 1 cigarette per day was estimated to account for $46 \%$ and 5 cigarettes per day for $57 \%$ of the increase in risk of developing coronary artery disease associated with a consumption of 20 cigarettes per day. These findings indicate an unexpectedly steep slope of exposure-harm relationship at low levels of consumption, with a relative levelling off at moderate-to-high levels. In terms of relative risk, estimated values for men and women consuming a single cigarette daily were 1.48 and 1.57 , respectively, whereas smoking 5 cigarettes per day was shown to carry a relative risk for coronary events of 1.58 and 1.76 in male and female subjects as compared to 2.04 and 2.84 for consumers of 20 cigarettes daily, respectively. Similar results were obtained from the analysis of stroke incidence with respect to the percentage of excess risk carried by smoking 1 to 5 cigarettes daily, although the respective values of relative risk were 
lower in each smoking and sex category, compared to coronary events.

The findings of this meta-analysis appear to provide even more robust evidence on the significant percentage of smoking-associated harm attributable to low-grade tobacco consumption, after considering that the investigators meticulously tried to exclude the possibility of overestimation of the light-smoking-associated relative risk, which could have been caused by classifying subjects who had recently reduced smoking as light smokers, thus ignoring the effect of longterm heavy tobacco consumption. In particular, based on the assumption that significant changes in smoking status would have most probably occurred in later years, studies with follow-up up to 1995 were selectively examined and the relative risks for heavy smokers (20 cigarettes daily) were compared to the respective values from the analysis of the total of studies. Because no significant differences were noted, the investigators concluded that a substantial bias resulting from misclassification of formerly heavy smokers cutting down on consumption was unlikely.

Taken together, these data leave little doubt about whether a safe level of chronic tobacco consumption exists and highlight that total abstinence should always be the ultimate goal. Howev- er, the aforementioned studies were not designed to assess the potential benefits from a harm-reduction strategy based on substantial reduction of tobacco consumption rates in cases where complete cessation is not feasible. Relevant evidence is summarized in a subsequent section.

\section{Potential mechanisms linking light smoking and cardiovascular disease}

The mechanisms by which smoking is causally related to the development of CVD are well established and include catecholamine release and ischaemia due to supply-demand mismatch, vasospasm, increased platelet reactivity, imbalance between prothrombotic and fibrinolytic activity, endothelial dysfunction, oxidative stress, activation of inflammation, impaired autonomic function, and insulin resistance [15, 16] (Figure 1). Studies suggest that several of these different effects of tobacco consumption are already evident at low consumption levels or even shortly after smoking a single cigarette. However, the exact reasons explaining the relative levelling off of the exposure-effect curve at moderate-to-high levels of consumption are still incompletely understood.

Theoretically, one could consider that the pathophysiological background of this non-linear relationship would have been adequately elucidated if

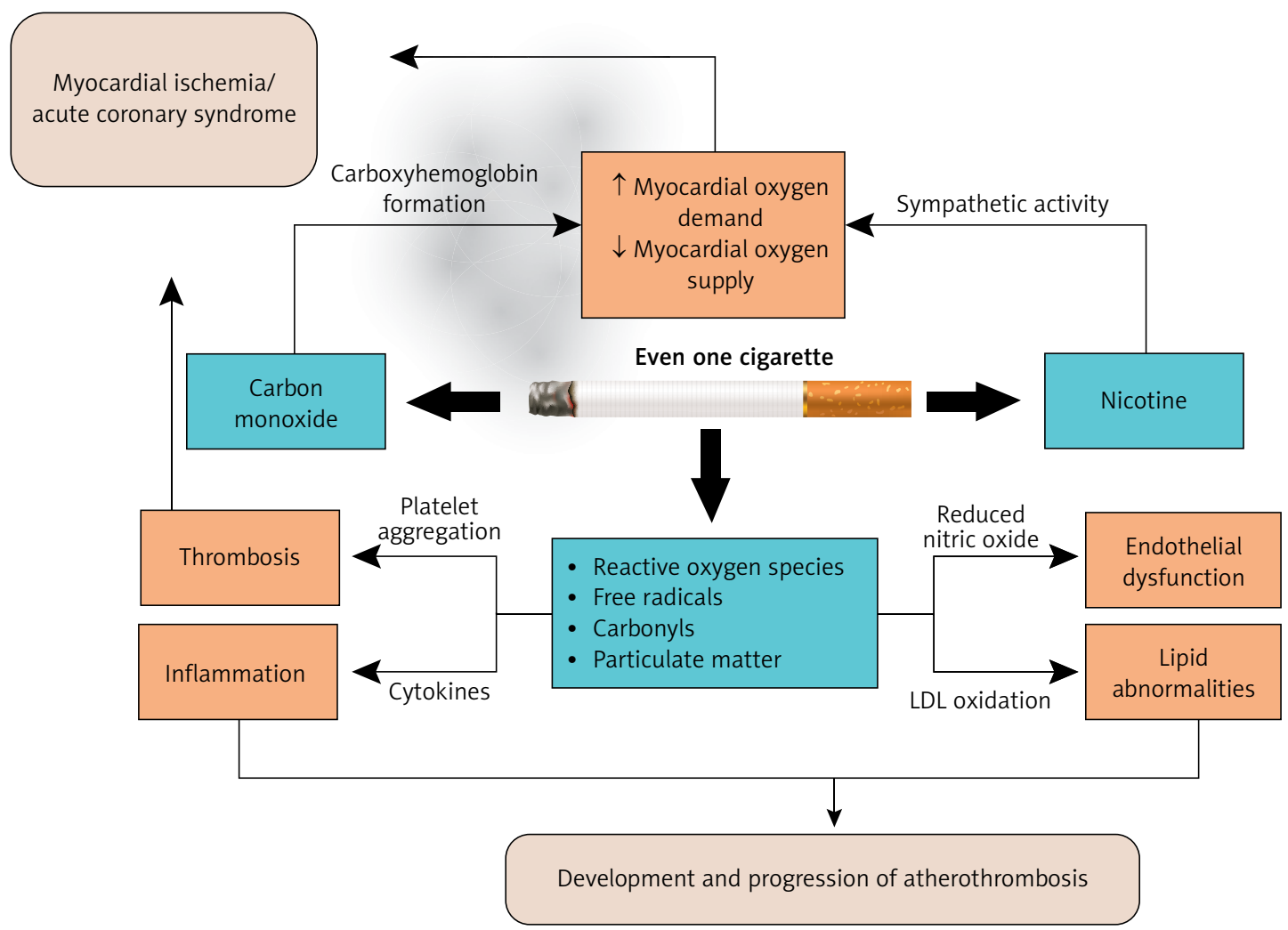

Figure 1. Pathophysiological principles of cardiovascular disease development in the case of light cigarette smoking: The potential mechanisms include catecholamine release and ischaemia due to supply-demand mismatch, vasospasm, increased platelet reactivity, imbalance between prothrombotic and fibrinolytic activity, endothelial dysfunction, oxidative stress, activation of inflammation, and impaired autonomic function 
there had been evidence that some of the biological effects of smoking already reach a peak at low tobacco doses, without further increases at higher levels - i.e. demonstrate a "saturation" effect. Unfortunately, there is a relative paucity of studies designed to evaluate the degree of smoking-induced pathophysiologic alterations regarding dose response; nevertheless, the measurable effects on coagulation parameters and vascular function observed after smoking a single cigarette provide clues in this direction and are worth mentioning. In addition, the effect of second-hand smoking on indices of platelet function, coagulation activity, endothelial function, and oxidative stress also provide indirect evidence, given the aforementioned comparable relative risks for cardiovascular events observed in subjects exposed to second-hand smoke and light smokers. It should be clarified, however, that composition and potential effects of "sidestream" smoke can differ considerably from that of "mainstream" smoke [17].

The effect of second-hand smoking on thrombotic status has been found to be significant, as has been demonstrated by Schmid et al. in a study assessing the effect of single and repeated exposure to second-hand smoke on the platelet reactivity profile of active smokers and non-smokers [18]. The evaluated indices of platelet activation included plasma thromboxane B2, 11-dehydro-thromboxane B2, malondialdehyde, as well as the conversion rate of arachidonic acid to thromboxane B2 and hydroxy-5, 8,10-heptadecatrienoic acid. Although the levels of these indices suggested a significantly higher platelet reactivity in smokers - as compared to non-smokers - both at baseline and after a single 60-minute exposure to second-hand smoke, no significant difference was found between the 2 groups of subjects after repeated exposure. The potentially detrimental effect of second-hand smoking - and therefore possibly of light active smoking, as well - on thrombotic status was also demonstrated in a Japanese study, in which passive-smoking-induced alterations in fibrinogen levels were found to be as high as $40-60 \%$ of the respective changes observed in active smokers [19].

The acute effect of smoking a single cigarette on platelet reactivity is highlighted in a study by Pamukcu et al., in which it was demonstrated that it can be strong enough to result in a change in the in vitro aspirin responsiveness status. Indeed, $25 \%$ of persistent smokers without baseline high on-treatment platelet reactivity were reclassified as "non-responders" 15 min after consuming their first cigarette [20].

Besides acutely affecting platelet activation and coagulation, smoking a single cigarette appears to also exert a remarkable adverse effect on arterial stiffness. Stefanadis et al. randomized 40 male ac- tive smokers undergoing diagnostic cardiac catheterization to either smoking or sham-smoking groups and subjected them to repeated assessment of aortic elastic properties by measurement of aortic pressure-diameter relationship, during the first $15 \mathrm{~min}$ post-intervention [21]. The investigators observed a significant deterioration in aortic elastic properties after smoking, which were maintained throughout the study period, as opposed to the lack of change in measurements in the sham-smoking group.

Vlachopoulos et al. investigated, through causeand-effect testing, whether acute smoking alters arterial stiffness in subjects who smoke on a chronic basis [22]. The novel finding of this study was that the deterioration of arterial elastic properties, which follows acute smoking, is dependent primarily on COX-1 activity and secondarily on COX-2 activity. This is because aspirin, a non-selective COX-1 and COX-2 inhibitor, fully abrogates the smoking-induced increase of pulse wave velocity (PWV), whereas celecoxib, a selective COX-2 inhibitor, only partially prevents the acute smoking-related adverse effect on PWV. The results of this study indicate that beyond the chronic arterial damage that accompanies chronic smoking, exacerbations of vascular dysfunction after smoking a single cigarette may contribute to the increased cardiovascular risk in chronic smokers.

The concept of acute deterioration in vascular status after smoking a single cigarette is further reinforced by a study conducted by MiYang et al., in which 38 nonsmokers and 29 smokers were enrolled, and whose blood vessel condition was assessed noninvasively by accelerated photoplethysmograph [23]. More precisely, measurements of blood vessel output power (BVOP), blood vessel ageing level (BVAL), blood vessel tension (BVT), and remained blood volume (RBV) were carried out in each subject at 2 different time points, designated as "pre-experiment" and "post-experiment", the latter 5 min after smoking a single cigarette in smokers. The findings of this study indicated a substantial acute deterioration in vascular status in smokers after consumption of a single cigarette; importantly, this effect could not be attributed to a change in $\mathrm{SpO}_{2}$, which did not occur. In addition, flow-mediated vasodilation, an established biomarker of endothelial function, has been shown to acutely decrease after smoking a single cigarette, along with indices of left ventricular function and arterial stiffness.

Taken together, these data indirectly suggest that the substantial effect of repeated low-grade exposure to tobacco smoke observed on platelet reactivity and coagulation profile, as well as early vascular dysfunction and haemodynamic impairment, could form part of the pathophysiological basis explaining the disproportionate contribution 
of low-grade tobacco consumption to increased cardiovascular risk. Conversely, this appears not to be the case for activation of inflammatory pathways, because markers of inflammation were demonstrated to exhibit a fairly predictable dose-response relation to quantity of cigarettes consumed in the Multiethnic Study of Atherosclerosis [24], which is - to our knowledge - the only study that directly examined levels of biomarkers reflecting the activity of a pathway known to be affected by smoking in relation to active smoking intensity.

\section{The effect of reducing the number of cigarettes on cardiovascular risk}

There are a relative paucity of data from clinical trials specifically designed to evaluate the effect of smoking reduction on cardiovascular outcomes. In this context, Song and Cho included 475,734 men aged 30-58 years, who were stratified into 9 groups by their smoking status at 2 different time points: at enrolment and 2 years later, and they were followed-up for 10 years [25]. The study endpoints were incidence of ischaemic and haemorrhagic stroke and myocardial infarction. A significant risk reduction was observed for myocardial infarction, subarachnoid haemorrhage, and ischaemic stroke in heavy smokers - consuming at least 20 cigarettes per day - who had quit smoking between the 2 time points of smoking status evaluation (hazard ratios: $0.43,0.58$, and 0.66 , respectively), compared to persistently heavy smokers. Conversely, in formerly heavy smokers who reduced cigarette consumption without quitting, only a trend towards decrease in risk of all stroke and myocardial infarction was observed, which did not reach statistical significance. Although the authors underline the need for further studies to elucidate the potentially harm-reducing effect of reducing cigarette consumption, the findings of this study imply that the aforementioned relatively flat dose-response curve at moderate-to-high, as opposed to low levels of exposure, is probably retained also in the reverse direction.

Similar results were obtained in the prospective cohort study of Godtfredsen et al. conducted in Denmark, which included 19,423 men and women in whom thorough information about their smoking status was obtained at 2 separate time points, 5 to 10 years apart [26]. Participants were followed-up from the time of second examination until first hospitalization for myocardial infarction (MI) or MI-associated death. Remarkably, because persistently heavy smokers were considered participants who continued to consume at least 5 cigarettes per day throughout the time interval between the 2 examinations, an unusually low threshold for defining heavy smoking. Where- as subjects who accomplished smoking cessation were shown to have a lower risk for Ml-associated hospitalization or death (hazard ratio $=0.71$ ), this was not the case in patients who had decreased smoking consumption by at least $50 \%$ by the time of the second assessment, without quitting (hazard ratio $=1.15$ ). The results of this study, which were preserved after adjustment for patients' clinical characteristics, further reinforce the concept of significant cardiovascular harm preservation from reducing smoking without quitting.

In an analogous prospective cohort study conducted in Norway, which aimed to determine the risk of death from any cause and from specific smoking-related diseases in heavy smokers of both sexes, who had reduced cigarette consumption by at least 50\%, Tverdal and Bjartveit enrolled 51,210 subjects aged 20-49 years in the mid 1970s. Participants were subsequently re-screened after 3 to 3 years and followed up until 2003 [27]. The findings of the study did not suggest a significant decrease in the risk of dying of any cause, CVD, smoking-related cancer, or lung cancer, underlining the importance of not generating false expectations of reduction in the risk of premature death in smoking-reducers who do not quit.

On the contrary, it is remarkable that both the Danish and the Korean groups of investigators reported a statistically significant decrease in the risk of lung cancer in formerly heavy smokers who reduced cigarette consumption by at least $50 \%$ or to less than 10 or even to 10-19 cigarettes per day $[28,29]$. These data once again imply fundamental differences between CVD and malignancies regarding the exact mechanisms by which tobacco exposure is implicated in the pathogenesis in each case.

\section{Temporary light smoking as a bridge to cessation}

While the adverse effects of persistent light smoking on cardiovascular health are now well established, and it is becoming clearer that a harm-reduction strategy based on consumption lowering cannot be encouraged, we feel it appropriate to re-emphasize the potentially dynamic character of an individual's smoking status. Given that complete abstinence from smoking is the ultimate goal in any case, a low level of cigarette consumption could also be also viewed from the perspective of increasing the likelihood of a successful cessation attempt. Indeed, in a large Korean study, CPD was found to be inversely related to smoking cessation success rates [30]. These data offer a more optimistic regard of low-grade cigarette consumption as compared to focusing on the unexpectedly high relative risk for major adverse coronary events. 
In a broader sense, a smoking-cessation strategy based on gradual reduction of consumption before eventually quitting can also be regarded as a sub-category of light smoking - albeit in a temporary sense. Whether such an approach increases the likelihood of a successful quitting attempt remains controversial and is the focus of ongoing research. In this context, in a Cochrane review published in 2012, the authors, primarily aiming to compare the success rates of gradual reduction and abrupt cessation quit-smoking strategies, analysed the results of 10 randomized controlled trials, including 3760 participants [31]. The primary outcome measure was complete 6-month abstinence from smoking at follow-up. There was a significant inter-study variability as for the implementation of behavioural or pharmacological support, but every pair of randomization arms in each study received the same degree and mode of support for their smoking cessation attempt. No significant difference of success rates between the 2 smoking cessation approaches were noted, and the mode of additional support provided in each case did not seem to influence the results, which suggests that a strategy based on temporary low-rate cigarette consumption immediately prior to cessation could be beneficial, at least in a subset of patients.

Conversely, the results of a randomized controlled trial conducted more recently by LindsonHawley et al. challenges the concept of equivalence between the 2 strategies. The study population consisted of 697 adult smokers who were randomized to be instructed to quit either abruptly or after gradually reducing cigarette consumption by $75 \%$ in the last 2 weeks prior to quitting [32]. Behavioural support, as well as nicotine replacement therapy, were provided in both groups. Validated abstinence from smoking 4 weeks after the day of quitting was defined as the primary endpoint and occurred in $49 \%$ of subjects randomized to the abrupt cessation group, whereas the success rate in the gradual cessation group was significantly lower, at $39.2 \%$. At 6 months, absistence rates were still higher in the abrupt cessation group, which, importantly, included subjects who initially preferred smoking cessation by gradual reduction.

In order to improve smoking cessation, motivational interviewing or counselling synergistically with pharmacotherapy are needed. Intensive counselling sessions seem to increase abstinence rates by $6.0 \%$. Whereas pharmacotherapy on top of intensive counselling doubles the cessation rate to $12.3 \%$. Nicotine replacement therapy, bupropion, and Varenicline are commonly used for smoking cessation. Public policy is also needed including smoking restrictions in public places and work places and campaigns for the awareness that there is no safe mode of smoking and the deleterious effects of light smoking [33].

In aggregate, these data suggest that an abrupt cessation strategy should be favoured in most cases, but one should always keep in mind that the population of smokers is highly heterogeneous with respect to the level of addiction and personality, and individualization is the cornerstone of any treatment approach.

\section{The cardiovascular risk in light smokers}

Despite the significant relative risk for major adverse cardiovascular events observed in smokers consuming as little as 1 to 5 CPD, it is the absolute - rather than the relative - cardiovascular risk that will ultimately dictate preventive and therapeutic decisions (besides smoking cessation) in everyday clinical practice. Current guidelines recommend stratification of patients into 1 of 5 categories of cardiovascular risk, namely low, low-to-moderate, moderate-to-high, high, and very high risk, based on models such as SCORE, which is based on estimation the 10-year probability of a fatal cardiovascular event, and the ACC/ AHA model, which aims to predict the incidence of both fatal and nonfatal events, by evaluating blood pressure value, total cholesterol level, age, sex, and smoking status $[34,35]$. Risk stratification is paramount in decision-making with respect to blood pressure management and lipid-lowering treatment, which are recommended to be more intense with higher estimated cardiovascular risk.

As mentioned above, smoking status is a continuous variable, but it should be emphasized that it is treated as a dichotomous one in both cardiovascular risk prediction models, which were based on patient cohorts that mostly included either heavy smokers or non-smokers [36]. The relative risk for cardiovascular events granted to smoking in these models is roughly 2 , with some variation according to baseline clinical characteristics, irrespective of the degree of tobacco consumption. In this context, it might be tempting to calculate the percentage of patients consuming 1 to 5 CPD, who would be reclassified to a lower risk category if the relative risk carried by their smoking status be downgraded from 2 to 1.4-1.6, according to the results of the landmark meta-analysis mentioned in a previous section. As illustrated in Figure 2, a light-smoking status defined as consuming 1-5 CPD would theoretically translate into less intensive lipid-lowering and antihypertensive therapy in a substantial proportion of 50-year-old, male, persistently light smokers, as compared to the scenario in which no distinction between light and heavy smokers is made. Whether such an approach would be appropriate, however, is highly doubtful, given the significant changes in tobac- 
A

10-year risk of fatal CV event in a 50-year old male smoker

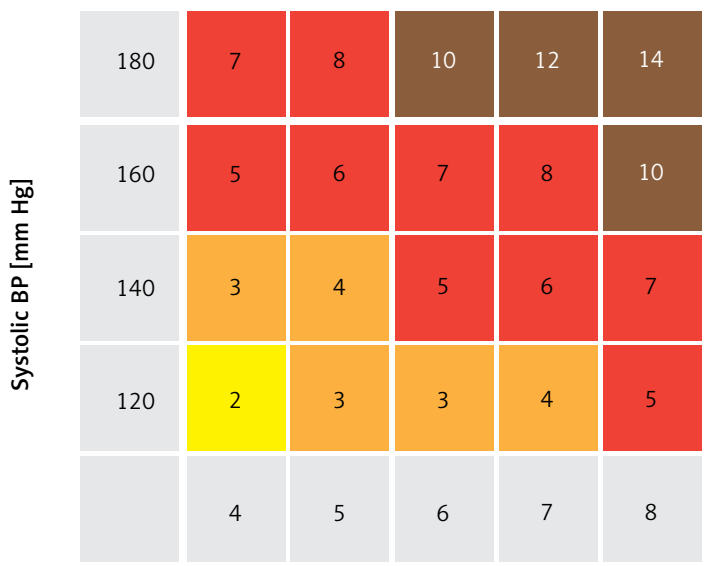

B

Theoretical 10-year risk of fatal CV event in a 50-year old male consuming 1-5 CPD

Total cholesterol [mmol/l]

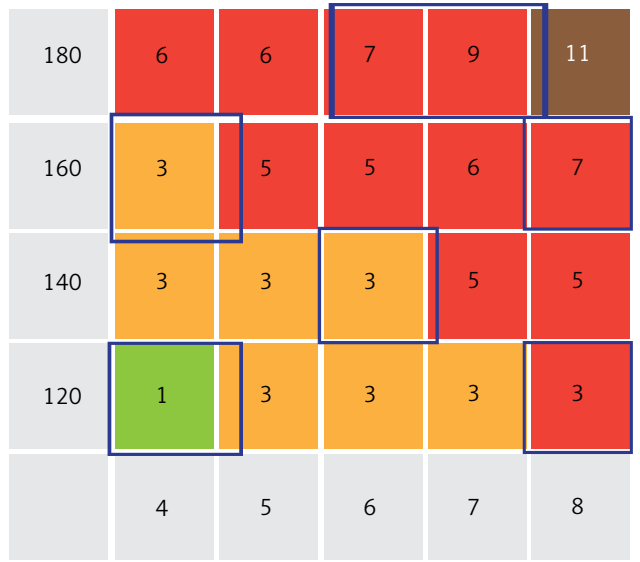

Figure 2. The theoretical effect of smoking 1 to 5 cigarettes per day on 10-year risk of development of a fatal cardiovascular event (\%) based on the SCORE risk prediction model and application of a relative risk value of 1.4-1.6, based on the respective relative risk values for this tobacco consumption category reported in recent studies and meta-analyses. A - Percentage risk of fatal cardiovascular event in a 50-year-old male smoker, as calculated by applying the SCORE model, by blood pressure and total cholesterol level category. B - Percentage risk for fatal cardiovascular event in a 50-year-old male light smoker (1-5 CPD), calculated by applying a relative risk value of 1.4-1.6 on the respective values for a non-smoker of the same gender and age (SCORE model). Colours: Green indicates a 0-1\% 10-year risk of fatal cardiovascular event, yellow indicates a 2\% 10-year risk, orange stands for a 3-4\% 10-year risk, red indicates a 5-9\% 10-year risk, and dark red stands for an absolute risk value of at least $10 \%$ (very-high-risk category). Blue squares in panel B demarcate reclassification to a lower-risk category, by applying a lower (1.4-1.6) relative risk value for consumption of 1-5 cigarettes per day, as opposed to smoking of unspecified intensity

$C V$ - cardiovascular, CPD-cigarettes per day.

co consumption rates over time, which could be upward rather than downward. Nevertheless, although this scenario of cardiovascular risk reclassification of light smokers is more of a hypothesis-generating rather than a truly evidence-based statement, we feel that it is worthwhile mentioning that aggressive lipid- and blood pressure-lowering strategies are not without risks.

\section{Conclusions}

Despite the fact that the pathophysiological mechanisms explaining the unexpectedly high degree of increase in cardiovascular risk carried by smoking as little as one CPD are still incompletely understood, the evidence clearly indicates that no safe level or mode of tobacco consumption exists. These findings are not in accordance with the risk of lung cancer, the incidence of which is dose dependent. The dissemination of this knowledge to the general population, and, perhaps more importantly, to persistent smokers at high cardiovascular risk or with established CVD, is important and should be part of everyday clinical practice. Brief advice and information on the disproportionately low benefit from cutting down tobacco consumption without quitting should be provided at every opportunity. However, clinicians should always bear in mind that congratulating patients for their effort in significantly reducing smoking - which in itself is an achievement - is not equal to generating misconceptions and could be a valuable aid in ultimately achieving the goal of quitting.

\section{Conflict of interest}

The authors declare no conflict of interest.

\section{References}

1. Nichols M, Townsend N, Scarborough P, Rayner M. Cardiovascular disease in Europe 2014: epidemiological update. Eur Heart J 2014; 35: 2950-9.

2. Hadley MB, Baumgartner J, Vedanthan R. Developing a clinical approach to mitigating risks of air pollution and protecting cardiovascular health. Circulation 2018; 137: 725-42.

3. Giovino GA, Mirza SA, Samet JM, et al.; GATS Collaborative Group. Tobacco use in 3 billion individuals from 16 countries: an analysis of nationally representative cross-sectional household surveys. Lancet 2012; 380: 668-79.

4. Hackshaw A, Morris JK, Boniface S, Tang JL, Milenković D. Low cigarette consumption and risk of coronary heart disease and stroke: meta-analysis of 141 cohort studies in 55 study reports. BMJ 2018; 360: j5855. 
5. Okuyemi KS, Harris KJ, Scheibmeir M, Choi WS, Powell J, Ahluwalia JS. Light smokers: issues and recommendations. Nicotine Tob Res 2002; 4 Suppl 2: S103-12.

6. Husten CG. How should we define light or intermittent smoking? Does it matter? Nicotine Tob Res 2009; 11: 111-21.

7. Jackson SE, Beard E, Kujawski B, et al. Comparison of trends in self-reported cigarette consumption and sales in England, 2011 to 2018. JAMA Netw Open 2019; 2: e1910161.

8. Jamal A, Homa DM, O'Connor E, et al. Current cigarette smoking among adults - United States, 2005-2014. MMWR Morb Mortal Wkly Rep 2015; 64: 1233-40.

9. Amrock SM, Weitzman M. Adolescents' perceptions of light and intermittent smoking in the United States. Pediatrics 2015; 135: 246-54.

10. Dela Cruz CS, Tanoue LT, Matthay RA. Lung cancer: epidemiology, etiology, and prevention. Clin Chest Med 2011; 32: 605-44.

11. Bjartveit $\mathrm{K}$, Tverdal A. Health consequences of smoking 1-4 cigarettes per day. Tobacco Control 2005; 14: 315-20.

12. Law MR, Morris JK, Wald NJ. Environmental tobacco smoke exposure and ischaemic heart disease: an evaluation of the evidence. BMJ 1997; 315: 973-80.

13. Pope CA 3rd, Burnett RT, Krewski D, et al. Cardiovascular mortality and exposure to airborne fine particulate matter and cigarette smoke: shape of the exposure-response relationship. Circulation 2009; 120: 941-8.

14. Prescott E, Scharling H, Osler M, Schnohr P. Importance of light smoking and inhalation habits on risk of myocardial infarction and all cause mortality. A 22 year follow up of 12149 men and women in The Copenhagen City Heart Study. J Epidemiol Community Health 2002; 56: 702-6.

15. U.S. Department of Health and Human Services. The health consequences of smoking: 50 years of progress. A report of the Surgeon General . Department of Health and Human Services, Centers for Disease Control and Prevention, Coordinating Center for Health Promotion, National Center for Chronic Disease Prevention and Health Promotion, Office on Smoking and Health 2014.

16. Reaven G, Tsao PS. Insulin resistance and compensatory hyperinsulinemia: the key player between cigarette smoking and cardiovascular disease? J Am Coll Cardiol 2003; 41: 1044-7.

17. Rubenstein D, Jesty J, Bluestein D. Differences between mainstream and sidestream cigarette smoke extracts and nicotine in the activation of platelets under static and flow conditions. Circulation 2004; 109: 78-83.

18. Schmid P, Karanikas G, Kritz H, et al. Passive smoking and platelet thromboxane. Thromb Res 1996; 81: 451-60.

19. Iso H, Shimamoto T, Sato S, Koike K, lida M, Komachi Y. Passive smoking and plasma fibrinogen concentrations. Am J Epidemiol 1996; 144: 1151-4.

20. Pamukcu B, Oflaz H, Onur I, Cimen A, Nisanci Y. Effect of cigarette smoking on platelet aggregation. Clin App Thromb Hemost 2011; 17: E175-80.

21. Stefanadis C, Tsiamis E, Vlachopoulos C, et al. Unfavorable effect of smoking on the elastic properties of the human aorta. Circulation 1997; 95: 31-8.

22. Vlachopoulos C, Aznaouridis K, Bratsas A, et al. Arterial stiffening and systemic endothelial activation induced by smoking: the role of COX-1 and COX-2. Int J Cardiol 2015; 189: 293-8.

23. MiYang J, HyeonCheol J, Lee K, Yim J. The acute effect of smoking a single cigarette on vascular status, $\mathrm{SpO} 2$, and stress level. Med Sci Monit 2014; 20: 601-7.
24. Al Rifai M, DeFilippis AP, McEvoy JW, et al. The relationship between smoking intensity and subclinical cardiovascular injury: the Multi-Ethnic Study of Atherosclerosis (MESA). Atherosclerosis 2017; 258: 119-30.

25. Song YM, Cho HJ. Risk of stroke and myocardial infarction after reduction or cessation of cigarette smoking: a cohort study in Korean men. Stroke 2008; 39: 2432-8.

26. Godtfredsen NS, Osler M, Vestbo J, Andersen I, Prescott E. Smoking reduction, smoking cessation, and in cidence of fatal and non-fatal myocardial infarction in Denmark 1976-1998: a pooled cohort study. J Epidemio Community Health 2003; 57: 412-6.

27. Tverdal A, Bjartveit K. Health consequences of reduced daily cigarette consumption. Tob Control 2006; 15: $472-80$

28. Godtfredsen NS, Prescott E, Osler M. Effect of smoking reduction on lung cancer risk. JAMA 2005; 294: 1505-10.

29. Song YM, Sung J, Cho HJ. Reduction and cessation of cigarette smoking and risk of cancer: a cohort study of Korean men. J Clin Oncol 2008; 26: 5101-6.

30. Yang JJ, Song M, Yoon HS, et al. What are the major determinants in the success of smoking cessation: results from the health examinees study. PLoS One 2015; 10: e0143303.

31. Lindson-Hawley N, Aveyard P, Hughes JR. Reduction versus abrupt cessation in smokers who want to quit. Cochrane Database Syst Rev 2012; 11: CD008033.

32. Lindson-Hawley N, Banting M, West R, Michie S, Shinkins B, Aveyard P. Gradual versus abrupt smoking cessation: a randomized, controlled noninferiority trial. Ann Intern Med 2016; 164: 585-92.

33. Wu J, Sin DD. Improved patient outcome with smoking cessation: when is it too late? Int J Chron Obstruct Pulmon Dis 2011; 6: 259-67.

34. Piepoli FM, Hoes AW, Agewall S, et al. ESC Scientific Document Group; 2016 European Guidelines on cardiovascular disease prevention in clinical practice: The Sixth Joint Task Force of the European Society of Cardiology and Other Societies on Cardiovascular Disease Prevention in Clinical Practice (constituted by representatives of 10 societies and by invited experts) Developed with the special contribution of the European Association for Cardiovascular Prevention \& Rehabilitation (EACPR), Eur Heart J 2016; 37: 2315-81.

35. Goff Jr DC, Lloyd-Jones DM, Bennett G, et al. 2013 ACC/ AHA Guideline on the Assessment of Cardiovascular Risk. A Report of the American College of Cardiology/ American Heart Association Task Force on Practice Guidelines. Circulation 2013; 129: S49-73.

36. Conroy RM, Pyörälä K, Fitzgerald AP, et al. Estimation of ten-year risk of fatal cardiovascular disease in Europe: the SCORE project. Eur Heart J 2003; 24: 987-1003. 